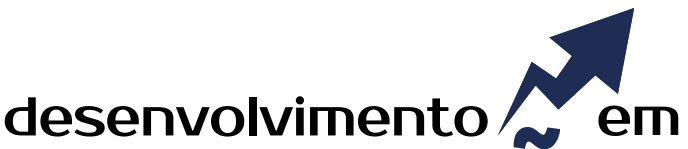 QUESTÂOO
}

\section{Quem é o Consumidor Sustentável? \\ Uma Revisão Sistemática da Literatura Internacional Sobre os Fatores Influenciadores do Consumo Sustentável}

http://dx.doi.org/10.21527/2237-6453.2020.54.104-122

Recebido em: $15 / 5 / 2020$

Aceito em: $24 / 11 / 2020$

\section{Arthur William Pereira da Silva ${ }^{1}$, Walid Abbas El-Aouar ${ }^{2}$, Eliana Andrea Severo ${ }^{3}$, Ahiram Brunni Cartaxo de Castro ${ }^{4}$, Cinthia Gabrielle Celedonio Silva ${ }^{5}$}

\begin{abstract}
RESUMO
Diversas pesquisas defendem que o conjunto dos principais fatores determinantes do comportamento de consumo sustentável ainda não é suficientemente claro, sendo necessários esforços adicionais que possibilitem avanços nesse sentido. Partindo dessa lacuna, o objetivo desta pesquisa foi identificar os principais fatores determinantes do consumo sustentável já identificados mundialmente. Este estudo foi desenvolvido por intermédio do método da revisão sistemática de literatura. Para tanto, foram definidos 7 termos de busca, pesquisados na base de dados Web of Science. Ao final das buscas e análises, compuseram a amostra de estudo 143 artigos. Por meio da revisão foram identificados 21 fatores que se destacam como determinantes do consumo sustentável, dentre os quais a consciência ambiental se sobressai. Vários fatores menos intuitivos ou evidentes, entretanto, também foram verificados. Por exemplo: acesso às redes sociais; nível de religiosidade; taxa de penetração do transporte público; amor pela posse material; taxa de urbanização; emoções, entre outros. Os resultados do estudo contribuem para a formação de uma abordagem teórica mais sólida para se analisar e entender o comportamento de consumo sustentável. Almeja-se, por meio disso, proporcionar às empresas um melhor delineamento dos fatores motivadores do consumo sustentável, que, em posse de tais informações, poderão desenvolver estratégias mais assertivas no atendimento das demandas desse público, potencializando suas vendas bem como contribuindo para a solidificação do desenvolvimento sustentável.
\end{abstract}

Palavras-chave: Marketing verde. Consumo verde. Marketing sustentável. Consumo consciente.

\section{WHO IS THE SUSTAINABLE CONSUMER? A SYSTEMATIC REVIEW OF THE INTERNATIONAL LITERATURE ON THE FACTORS INFLUENCING SUSTAINABLE CONSUMPTION}

\begin{abstract}
Several researches argue that the set of main factors determining the behavior of sustainable consumption is still not sufficiently clear, and additional efforts are needed to make progress in this direction. Starting from this gap, the objective of this research was to identify the main determinants of sustainable consumption already identified worldwide. This study was developed using the systematic literature review method. For this purpose, 7 search terms were defined, searched in the Web of Science database. At the end of the searches and analyzes, 143 articles were included in the study sample. Through the review, 21 factors were identified that stand out as determinants of sustainable consumption, among which environmental awareness stands out. However, several less intuitive or evident factors were also verified. For example: Access to social networks; Level of religiosity; Public transport penetration rate; Love for material possession; Urbanization rate; Emotions, among others. The results of the study contribute to the formation of a more solid theoretical approach to analyze and understand sustainable consumption behavior. The aim is thereby to provide companies with a better outline of the motivating factors for sustainable consumption, which in possession of such information, will be able to develop more assertive strategies in meeting the demands of this public, boosting their sales, as well as contributing to the solidification of development sustainable.
\end{abstract}

Keywords: Green Marketing. Green consumption. Sustainable Marketing. Conscious Consumption.

\footnotetext{
${ }^{1}$ Autor correspondente. Instituto Federal do Ceará (IFCE). Av. Jorge Dumar, 1703 - Jardim América. CEP 60410-426. Fortaleza/Ceará, Brasil. http://lattes.cnpq.br/3469488067. http://orcid.org/0000-0002-4515-6581813540. arthur.silva@ifce.edu.br

2 Universidade Potiguar (UNP). Natal/RN, Brasil.

${ }^{3}$ Universidade Federal de Pernambuco (UFPE). Recife/PE, Brasil.

${ }^{4}$ Instituto Federal de Educação, Ciência e Tecnologia do Rio Grande do Norte (IFRN). Natal/RN, Brasil.

${ }^{5}$ Instituto Federal do Ceará (IFCE). Jaguaruana/CE, Brasil.
} 
Apesar das diversas ações já empreendidas para promover o desenvolvimento sustentável em todo o mundo, o Painel Intergovernamental sobre Mudança Climática (IPCC) continua identificando crescimento na ocorrência e nível dos eventos ligados às condições de clima extremo nas últimas décadas (furacões, inundações, ondas de calor e secas), eventos estes causados pelas externalidades negativas do modo de produção hegemônico nas sociedades humanas, que geram vários prejuízos aos homens e aos demais seres vivos, causando, por exemplo, mudanças nocivas aos ecossistemas, perdas estruturais, danos à saúde e, em última instância, a morte (SOMERVILLE; WILLIAMS, 2015; IPCC, 2014; SILVA; SIQUEIRA, 2015; SILVA, B. G. et al., 2018; SILVA, A. W. P. et al., 2018; COELHO, C. et al., 2018).

O rápido desenvolvimento econômico experimentado nas últimas décadas trouxe consigo um conjunto de problemáticas ambientais e sociais ao planeta, além de agravar as já existentes (IPCC, 2014; SOMERVILLE; WILLIAMS, 2015; JOSHI; RAHMAN, 2017; FIGUEROA-GARCÍA; GARCÍA-MACHADO; YÁBAR, 2018; SILVA et al., 2019).

Uma das principais ações para reverter essa problemática socioambiental e solidificar o desenvolvimento sustentável é o fomento do consumo sustentável, uma vez que este tipo de comportamento visa a um processo de consumo que minimiza as externalidades negativas ao meio ambiente e à sociedade (ÜLKÜ; HSUAN, 2017; KHAN et al., 2018; COELHO, A. L. A. L. et al., 2018a; BRITO et al., 2019; COELHO, A. L. A. L. et al., 2018b).

Vale salientar que, além de fundamental para a promoção do desenvolvimento sustentável, o fomento ao consumo sustentável também é benéfico e atrativo para as empresas, uma vez que o aumento do número desse tipo de consumidor representa o crescimento de um nicho de mercado que tem sido alvo de cada vez mais empresas nas últimas décadas, em virtude da sua já notória representatividade.

Partindo dessa problemática, percebe-se a importância da promoção do comportamento de consumo sustentável, sendo ainda muito necessário, para tanto, melhor definir o conjunto dos principais fatores determinantes desse comportamento, uma vez que ainda não existem pesquisas suficientes nesse sentido (FIGUEROA-GARCÍA; GARCÍA-MACHADO; YÁBAR, 2018; SEMPREBON et al., 2019; SUN; LIU; ZHAO, 2019). Surge, assim, a questão central desta pesquisa: Quais os principais fatores determinantes do comportamento de consumo sustentável? Isso, por sua vez, levou ao objetivo do estudo: identificar os principais fatores determinantes do consumo sustentável já identificados mundialmente.

Por meio de revisão inicial da literatura internacional foi possível identificar que vários pesquisadores (BECK; PEREIRA, 2012; SILVA; SILVEIRA-MARTINS; OTTO, 2017; FIGUEROA-GARCÍA; GARCÍA-MACHADO; YÁBAR, 2018; SUN; LIU; ZHAO, 2019) entendem que o conjunto dos principais fatores determinantes do comportamento de consumo sustentável ainda não é suficientemente claro, sendo necessários esforços adicionais que possibilitem avanços nesse sentido, como o presente estudo.

Indicando a necessidade de aperfeiçoar a teoria preditiva do comportamento de consumo sustentável, Beck e Pereira (2012, p. 74) afirmaram taxativamente que ainda não existem estudos ou teorias capazes de explicar de forma satisfatória o comportamento de consumo consciente, deixando clara a necessidade da realização de mais estudos que visem a contribuir com a solidificação dessa teoria, como a presente pesquisa. 
Silva, Silveira-Martins e Otto (2017, p. 75) complementam sugerindo que construtos potencialmente influenciadores do comportamento de consumo sustentável, propostos por diferentes pesquisadores, poderiam ser estudados em conjunto para melhor se compreender esse complexo comportamento de consumo.

Figueroa-García, García-Machado e Yábar (2018) afirmam ser provável que muitos construtos relevantes para a explicação do comportamento de consumo sustentável não tenham sequer sido considerados nos modelos explicativos propostos, argumentando, ainda, que é fundamental avançar na identificação dessas variáveis e produzir escalas para mensurá-las.

Por fim, Sun, Liu e Zhao (2019) asseveram que, até onde os autores puderam investigar, nenhum modelo teórico demonstrou ter considerado todos os fatores relevantes do consumo sustentável até o momento, e que o foco das pesquisas futuras deve ser melhorar os modelos explicativos propostos até o momento, dando-lhes mais robustez.

Tais proposições evidenciam a contribuição teórica desta pesquisa, qual seja, contribuir para a formulação de uma teoria mais sólida a partir da qual se possa explicar e compreender melhor o comportamento dos consumidores sustentáveis por meio da proposição de um framework integrador que reúna as principais características desses indivíduos.

Alguns estudos já objetivaram a realização de esforços semelhantes aos desta pesquisa, como o realizado por Bamberg e Möser (2007) e publicado no Journal of Environmental Psychology, importante periódico da área de psicologia em âmbito global, bem como, e mais recentemente, a pesquisa realizada por Semprebon et al. (2019) e publicada no Journal of Marketing Intelligence \& Planning, que figura entre os mais relevantes da área de marketing.

Apesar, entretanto, da robustez metodológica e da notória contribuição de ambas as pesquisas, alguns fatores limitantes sugerem a necessidade de novos estudos com 0 mesmo intuito, sendo estes, primeiramente, a questão temporal, levando em consideração que o estudo desenvolvido por Bamberg e Möser (2007) foi publicado há mais de dez anos, e o estudo de Semprebon et al. (2019), que, apesar de ter sido publicado já em 2019, levou em conta em sua revisão de literatura apenas artigos editados de 2000 a 2016, oportunizando a realização de novos estudos que observem as pesquisas e artigos sobre o tema nos últimos três anos, 2017-2019.

Em segundo lugar, outro fator limitador dos resultados da pesquisa realizada por Semprebon et al. (2019) reside na consideração de artigos oriundos apenas das revistas da área de negócios e marketing, classificadas pelo extrato Q1 do SCImago Journal \& Country Rank, para a composição da sua amostra, fato que não desmerece em nada o estudo, que traz grande contribuição à área, porém enseja estudos complementares, como o presente, que leve em conta artigos provindos de revistas de diferentes áreas, além da área da administração e marketing, como da psicologia, ciências ambientais e até mesmo da nutrição, por exemplo, pois, como o tema é demasiado interdisciplinar e transversal, é tratado em estudos provenientes de diferentes áreas, e, consequentemente, publicado em revistas de áreas distintas, como no caso da pesquisa desenvolvida por Bamberg e Möser (2007), publicada em importante periódico da área de psicologia. 
Outro diferencial da presente pesquisa em relação à investigação realizada por Semprebon et al. (2019), é que esta, além de considerar artigos provindos de diferentes áreas, utilizou um critério diferente para a definição das revistas das quais se selecionou os artigos que compuseram a amostra de estudo. Enquanto a pesquisa de Semprebon et al. (2019) focou nos artigos publicados em revistas classificadas pelo extrato Q1 do SClmago Journal \& Country Rank, métrica de avaliação de periódicos atrelada à base Scopus, este estudo considerou os artigos publicados na coleção principal da Web of Science, base de dados internacional tão relevante quanto a Scopus, denotando o caráter complementar entre os estudos.

Além da contribuição teórica, por meio dos resultados desta pesquisa também almejou-se entregar aos profissionais de mercado uma melhor definição das características do público-alvo do segmento do consumo sustentável, baseada nos diversos fatores determinantes do consumo sustentável aqui identificados, a fim de sintetizar tais informações e, assim, possibilitar uma melhor orientação para balizar as estratégias das empresas que pretendem ou já investem nesse segmento. Segundo Silva, Silveira-Martins e Otto (2017) e Minton et al. (2018), quanto mais bem uma organização conhecer os fatores determinantes do consumo sustentável, mais eficientes tendem a ser suas estratégias de marketing ambiental.

\section{METODOLOGIA}

O método de pesquisa adotado para alcançar o objetivo desta pesquisa foi a revisão sistemática de literatura, que teve como intuito identificar os principais fatores determinantes do consumo sustentável já identificados e validados estatisticamente em âmbito global, tendo sido desenvolvida da seguinte forma: Primeiro foram definidos os termos de busca para operacionalizar a pesquisa. Os termos de busca definidos foram: "Consumo" AND "sustentável"; "consumo" AND "consciente"; "consumo" AND "ecológico" "consumo" AND "responsável"; "consumo" AND "verde"; "consumo" AND "ecologicamente" AND "consciente". Esta seleção de termos de busca ocorreu tendo como critério a utilização da maior quantidade conhecida de termos similares ao termo central estudado nesta pesquisa - consumo sustentável - para, assim, minimizar a perda de artigos relevantes para o escopo da análise proposta. O conhecimento de tais termos similares, por sua vez, deu-se pela leitura dos diversos artigos retornados na busca com a utilização do primeiro termo de busca: “Consumo" AND "sustentável". Depois os termos foram traduzidos para o inglês, tendo em vista ser essa a língua que predomina no universo científico, e, por conseguinte, na qual a maioria dos manuscritos relevantes são escritos e publicados. Foram, então, traduzidos da seguinte forma: "Consumption" AND "sustainable"; "Consumption" AND "conscious"; "Consumption" AND "ecological" "consumption" AND "responsible"; "Consumption" AND "green"; "Consumption" AND "ecologically" AND "conscious".

Em seguida foi definida a base de dados por meio da qual foi operacionalizada a busca dos manuscritos: a base Web of Science, mantida pela Clarivate Analytics. A escolha por tal base deu-se por esta ser uma das maiores e mais abrangentes bases de indexação de artigos e demais manuscritos científicos do mundo, cobrindo, de acordo com Moura et al. (2017) e Castro et al. (2018), mais de cem áreas do conhecimento científico, sendo, assim, uma das bases de dados mais extensas, bem como pelo alto nível de exigência que esta impõe às revistas que desejam se vincular a ela, pressupondo qualidade e relevância às pesquisas nela indexadas. 
Após a escolha da base foi realizada a procura pelos artigos por meio da pesquisa dos termos de busca definidos inicialmente. Quanto aos filtros disponíveis na Web of Science para o refinamento da pesquisa, utilizou-se apenas o filtro para que só retornassem manuscritos nos quais os termos de busca apareciam nos títulos, tendo em vista que nas primeiras buscas, onde tal filtro não foi aplicado, retornou um volume impossível de ser analisado, bem como uma quantidade demasiada de trabalhos fora do escopo que se objetivava com a revisão.

Inicialmente retornaram 1.874 artigos relacionados aos termos pesquisados, porém foi feita uma análise dos títulos e resumos de cada artigo a fim de verificar-se a ligação destes com a temática específica em análise: fatores determinantes do comportamento de consumo sustentável. Ao final desta triagem foram identificados e compuseram a amostra a ser mais profundamente analisada 143 artigos.

Por meio da leitura completa dos 143 artigos que passaram pela triagem inicial, identificaram-se os principais fatores determinantes do comportamento de consumo sustentável propostos até os dias atuais, testados e validados, que são apresentados no tópico seguinte deste trabalho.

\section{RESULTADOS E DISCUSSÃO}

Neste tópico é apresentado o resultado da revisão sistemática de literatura, composto por uma síntese que, por si só, já se mostra valiosa para as corporações, tendo em vista sua relevância para nortear as decisões e estratégias de marketing sustentável destas, uma vez que conglomera as principais características dos consumidores sustentáveis.

O Quadro 1 apresenta a síntese dos fatores determinantes do consumo sustentável já testados e validados e identificados na revisão de literatura.

Quadro 1 - Principais fatores determinantes do consumo sustentável identificados

\begin{tabular}{|c|c|}
\hline Fatores determinantes & Autores/ano \\
\hline \multirow{17}{*}{ Consciência ou preocupação Ambiental } & Stone et al. (1995) \\
\hline & Roberts (1996) \\
\hline & Lages e Vargas Neto (2002) \\
\hline & Gonçalves-Dias, Teodósio e Carvalho (2009) \\
\hline & Silva, Silveira-Martins e Otto (2017) \\
\hline & Ribeiro e Veiga (2011) \\
\hline & De Young (2000) \\
\hline & Straughan e Roberts (1999) \\
\hline & Colares e Mattar (2016) \\
\hline & Figueroa-García, García-Machado e Yábar (2018) \\
\hline & Chan e Lau (2000) \\
\hline & Latif et al. (2013) \\
\hline & Zareie e Navimipour (2016) \\
\hline & García et al. (2003) \\
\hline & Semprebon et al. (2019) \\
\hline & Bedante e Slongo (2004) \\
\hline & Sun, Liu e Zhao (2019) \\
\hline \multirow[t]{2}{*}{ Percepção de compromisso social } & Marquina e Morales (2012) \\
\hline & Wang et al. (2019) \\
\hline
\end{tabular}




\begin{tabular}{|c|c|}
\hline \multirow[t]{2}{*}{ Personalidade } & Fraj e Martinez (2006) \\
\hline & Semprebon et al. (2019) \\
\hline \multirow{5}{*}{ Emoções } & Fraj e Martinez (2007) \\
\hline & Semprebon et al. (2019) \\
\hline & Antonetti e Maklan (2016) \\
\hline & Brengman, Willems e Joye (2012) \\
\hline & Maloney, Ward e Braucht (1975) \\
\hline Altruísmo & Straughan e Roberts (1999) \\
\hline \multirow{2}{*}{$\begin{array}{l}\text { Efetividade do comportamento ambiental per- } \\
\text { cebida pelo consumidor }\end{array}$} & Straughan e Roberts (1999) \\
\hline & Sun, Liu e Zhao (2019) \\
\hline \multirow[t]{2}{*}{ Ativismo político } & Gonçalves-Dias, Teodósio e Carvalho (2009) \\
\hline & Espejo e Vázquez (2017) \\
\hline Orientação política de esquerda & Straughan e Roberts (1999) \\
\hline Planejamento de compra & Karmarkar e Bollinger (2015) \\
\hline Exposição a produtos verdes & Mazar e Zhong (2010) \\
\hline \multirow{6}{*}{ Atitudes ambientais } & Sun, Liu e Zhao (2019) \\
\hline & Tarkiainen e Sundqvist (2005) \\
\hline & Fielding, McDonald e Louis (2008) \\
\hline & Chen, Yeh e Wang (2008) \\
\hline & Paul, Modi e Patel (2016) \\
\hline & Semprebon et al. (2019) \\
\hline Estresse ambiental & Sun, Liu e Zhao (2019) \\
\hline Taxa de urbanização & Sun, Liu e Zhao (2019) \\
\hline Taxa de penetração do transporte público & Sun, Liu e Zhao (2019) \\
\hline Poluição ambiental & Sun, Liu e Zhao (2019) \\
\hline Tendência ambiental & Sun, Liu e Zhao (2019) \\
\hline \multirow{4}{*}{ Nível de religiosidade } & Minton et al. (2018) \\
\hline & Mathras et al. (2016) \\
\hline & Minton e Kahle (2016) \\
\hline & Razzaq et al. (2018) \\
\hline Amor à posse material & Dong et al. (2018) \\
\hline Influência da família e dos amigos & Figueroa-García, García-Machado e Yábar (2018) \\
\hline Condições de mercado & Figueroa-García, García-Machado e Yábar (2018) \\
\hline Acesso às redes sociais & Lien, Cao e Zhou (2017) \\
\hline
\end{tabular}

Fonte: Elaboração própria, 2019.

A seguir são apresentadas e discutidas as características de cada fator, como se dá a relação de causalidade entre os fatores e o comportamento de consumo sustentável, bem como é feita a conexão entre fatores e sua importância prática para as estratégias de marketing das empresas.

\section{Consciência ou Preocupação Ambiental}

Dentre o conjunto de fatores determinantes do consumo sustentável já testados e identificados, destacadamente o fator mais pesquisado, e apontado como principal influenciador desse tipo de consumo, é a consciência ambiental, ou a preocupação com o meio ambiente. Tal fator foi identificado como componente dos modelos preditivos em cerca de 16 estudos diferentes, como evidenciado no Quadro 1; estudos estes realizados com amostras do ocidente (FIGUEROA-GARCÍA; GARCÍA-MACHADO; YÁBAR, 2018) e do oriente (SUN; LIU; ZHAO, 2019), com ampla heterogeneidade em relação às características dos indivíduos que as compuseram. 
A consciência ambiental refere-se à forma com que um indivíduo percebe seu papel ante as questões ambientais (SILVA; SILVEIRA-MARTINS; OTTO, 2017). Também pode ser entendida como o posicionamento dos indivíduos perante as problemáticas do meio ambiente, ou seja, alguém que se posiciona de forma altamente comprometida com as questões ambientais possui um alto nível de consciência ambiental, bem como é verdadeira a relação inversa (BEDANTE; SLOGON, 2004).

\section{Percepção de Compromisso Social}

A percepção de compromisso social também foi identificada nos resultados de duas pesquisas (MARQUINA; MORALES, 2012; WANG et al., 2019) como sendo um fator determinante para o comportamento de consumo sustentável, apresentando relação preditiva positiva e significativa para com o construto dependente em análise, posto que quanto maior a percepção de compromisso social que os consumidores tenham em relação à determinada empresa, maior a disposição para praticar o comportamento de consumo sustentável.

Com base na teoria da troca social, os comportamentos de consumo sustentáveis são considerados reciprocidade a favor do provedor de serviços que demonstra compromisso com o social, ou seja, quanto maior percepção de compromisso social os indivíduos tiverem em relação a uma empresa, maior a tendência de recompensá-la consumindo seus produtos sustentáveis (WANG et al., 2019).

\section{Personalidade}

Outro fator que apresentou relação de predição positiva e significativa para com o construto comportamento de consumo sustentável foi a variável personalidade (FRAJ; MARTINEZ, 2006). Características de personalidade dos indivíduos, como extroversão, agradabilidade, conscienciosidade, estabilidade emocional e intelecto ou imaginação, influenciam de forma significativa o comportamento ecológico do consumidor, de forma que foi identificado que indivíduos com traços de personalidade mais voltados à extroversão e agradabilidade são os mais propensos ao consumo sustentável (FRAJ; MARTINEZ, 2006).

Dessa forma, as empresas devem concentrar-se naquelas pessoas que são caracterizadas por atributos de personalidade, como extroversão e agradabilidade, a fim de persuadi-los a adquirir seus produtos sustentáveis (FRAJ; MARTINEZ, 2006).

\section{Emoções}

No ano seguinte, Fraj e Martinez (2007) realizaram outro estudo que forneceu evidência estatística significativa que reforçou o que estudos bem mais antigos (MALONEY; WARD; BRAUCHT, 1975) já tinham identificado: as emoções são um fator determinante do comportamento de consumo sustentável, também devendo ser considerado pelas empresas e demais instituições na caracterização do seu público-alvo.

As duas pesquisas evidenciaram uma relação preditiva positiva e significativa das emoções para com o comportamento de consumo sustentável. De acordo com Fraj e Martinez (2007), as pessoas com maior grau de afeto ao ambiente terão uma atitude ambiental mais forte. 
Ainda quanto à influência das emoções sobre o comportamento de consumo sustentável, estudos indicam que a inveja influencia as atitudes dos consumidores, aumentando o desejo por produtos verdes, de forma que quanto maior o nível de inveja de um consumidor maior sua predisposição à compra de produtos verdes (ANTONETTI; MAKLAN, 2016).

A influência das emoções sobre o comportamento de consumo sustentável indica a importância do desenvolvimento de campanhas de marketing que promovam um maior afeto das pessoas para com o meio ambiente, a fim de ampliar o contingente de consumidores sustentáveis.

\section{Altruísmo}

O altruísmo também se mostrou um preditor positivo e significante estatisticamente do comportamento de consumo sustentável (STRAUGHAN; ROBERTS, 1999). Em seu estudo, Straughan e Roberts (1999) definem o altruísmo como o nível de preocupação de um indivíduo pelo bem-estar dos outros, e, a partir dessa definição e dos resultados do teste de seu modelo, os autores propõem que quanto maior o nível de altruísmo de alguém maior a propensão à prática do consumo sustentável.

\section{Efetividade do Comportamento Ambiental Percebida pelo Consumidor}

No mesmo estudo, outro fator considerado no modelo proposto deu evidência de ser um preditor positivo e significativo do comportamento de consumo sustentável, sendo este a efetividade do comportamento ambiental percebida pelo consumidor. Após 20 anos, Sun, Liu e Zhao (2019), ao inserir tal fator mais uma vez em um modelo de predição do consumo sustentável, obtiveram evidência estatística que vai ao encontro do indicado por Straughan e Roberts (1999) ainda no final do século 20.

A efetividade do comportamento ambiental, percebida pelo consumidor, refere-se ao nível de entendimento sobre se o que fazemos como indivíduos faz a diferença (STRAUGHAN; ROBERTS, 1999; SUN; LIU; ZHAO, 2019). Nas palavras de Sun, Liu e Zhao (2019, p. 484), o fator deve ser entendido como "a medida da percepção de um indivíduo sobre o grau de dificuldade de praticar o consumo verde e a confiança que essa pessoa tem em seu próprio consumo verde", de forma que quanto maior o nível dessa percepção maior a propensão ao consumo sustentável (STRAUGHAN; ROBERTS, 1999).

A validação dessa variável como preditora do consumo sustentável indica que não basta um posicionamento ambientalmente favorável por parte das organizações a fim de conquistar os consumidores sustentáveis, mas é necessário proporcionar a esses, por meio das ações de marketing, a percepção mais clara possível de que seu comportamento de consumo sustentável se converterá em benefícios reais para o meio ambiente. Nesse sentido, Straughan e Roberts (1999, p. 568) afirmam, baseados nos resultados de sua pesquisa, que 
Esforços de marketing baseados no meio ambiente devem estar explicitamente ligados a resultados benéficos. Aqueles atrelando simplesmente os produtos ao estereótipo "verde" já não são suficientes. Em vez disso, os profissionais de marketing devem mostrar como os consumidores, escolhendo produtos verdes, estão ajudando na luta para preservar o meio Ambiente.

\section{Ativismo Político}

Foram identificadas evidências estatísticas de que a probabilidade ou propensão de alguém se tornar um consumidor consciente está relacionada diretamente e, de forma positiva, à participação política ou ao ativismo político desses consumidores (GONÇALVES-DIAS; TEODÓSIO; CARVALHO, 2009; ESPEJO; VÁZQUEZ, 2017). Dessa forma, indica-se o ativismo político como mais um preditor significativo do comportamento de consumo sustentável.

Os consumidores sustentáveis convertem seus comportamentos de consumo em atitudes políticas, tanto "por meio de suas ações individuais como pela participação em movimentos sociais, transformando o mercado em uma arena pública e uma área para a prática da democracia." (ESPEJO; VÁZQUEZ, 2017, p. 59).

Sendo assim, os resultados dos estudos de Gonçalves-Dias, Teodósio e Carvalho (2009) e Espejo e Vázquez (2017) apontam o nível de ativismo político dos indivíduos como mais uma característica diferenciadora dos consumidores sustentáveis, de forma que quanto mais ativa politicamente for uma pessoa, maior sua propensão ao consumo sustentável. Isso deve deixar as empresas atentas ao fato de que o consumo de produtos e serviços por este público vai além da mera satisfação das suas necessidades pela utilidade prática do bem em si, mas, nessa "arena pública" (ESPEJO; VÁZQUEZ, 2017, p. 59), a aquisição e o uso de determinados produtos ofertados por uma empresa, em detrimento de seus similares oferecidos por suas concorrentes, funciona como símbolo de uma manifestação política em defesa de um ideal, no caso o ideal da sustentabilidade defendido pela empresa escolhida.

\section{Orientação Política de Esquerda}

Straughan e Roberts (1999) validaram ainda outro fator como sendo um preditor significativo do comportamento de consumo sustentável, sendo este a orientação política de esquerda.

É importante deixar clara a diferença entre este fator, validado por meio do modelo proposto por Straughan e Roberts (1999), e o fator apresentado nos parágrafos anteriores, o ativismo político, proposto e validado por Gonçalves-Dias, Teodósio e Carvalho (2009) e Espejo e Vázquez (2017). Enquanto o fator ativismo político indica que quanto mais ativo politicamente for um consumidor mais propenso ao consumo sustentável ele será, o fator orientação política de esquerda sinaliza que quanto maior for o nível de identificação de um consumidor com crenças políticas de esquerda, maior será a sua propensão ao consumo sustentável. 
Denota-se que, além de mais ativos politicamente, os consumidores sustentáveis são mais adeptos às crenças e ideologias de esquerda. Este é mais um importante direcionador das ações de marketing voltadas à promoção da imagem da empresa e dos seus produtos e serviços, indicando que muito cuidado deve ser tomado quanto ao embasamento político/ideológico da sua atuação.

\section{Planejamento de Compra}

Karmarkar e Bollinger (2015) obtiveram evidências de uma relação preditiva positiva entre o fator planejamento de compra e o comportamento de consumo sustentável. Ou seja, de acordo com os autores, os consumidores sustentáveis planejam suas compras. Ou, ainda, quanto mais planejados forem os comportamentos de consumo, mais sustentáveis eles tendem a ser.

Os resultados do estudo de Karmarkar e Bollinger (2015) demonstraram, por exemplo, que levar as próprias sacolas ao supermercado aumenta não apenas as compras de alimentos orgânicos, mas também de alimentos indulgentes.

\section{Exposição a Produtos Verdes}

Foi identificado por Mazar e Zhong (2010) em seus estudos como mais um fator influenciador do comportamento de consumo sustentável. De acordo com os pesquisadores, a mera exposição a produtos verdes ativa o senso de responsabilidade social e comportamento ético dos consumidores, ou seja, quanto maior o nível de exposição a tais produtos maior a tendência ao comportamento sustentável do consumidor.

De acordo com este estudo, as organizações que focam nesse segmento devem desenvolver estratégias de marketing, principalmente de promoção e distribuição, que possibilitem a maior experiência de contato possível entre o seu público-alvo e os seus produtos e serviços sustentáveis.

\section{Atitudes Ambientais}

O fator foi validado por meio de vários estudos como sendo um preditor significativo do consumo sustentável (TARKIAINEN; SUNDQVIST, 2005; FIELDING; MCDONALD; LOUIS, 2008; CHEN; YEH; WANG, 2008; PAUL; MODI; PATEL, 2016; SUN; LIU; ZHAO, 2019; SEMPREBON et al., 2019).

Dessa forma, os consumidores sustentáveis que não só apresentam um alto nível de consciência ambiental ou preocupação ambiental, mas que praticam atitudes ambientais favoráveis, tendem a apresentar níveis mais altos de comportamento de consumo sustentável. Tal constatação abre espaço para ações promovidas pelas empresas ou pelo governo que possibilitem e fomentem a prática de atitudes ambientais por parte dos indivíduos, práticas essas não necessariamente de consumo, mas simplesmente pró-ambientais, no intuito de ampliar a prática do consumo sustentável.

\section{Estresse Ambiental}

Este fator foi validado por meio do teste do modelo preditivo de Sun, Liu e Zhao (2019) como um fator influenciador significativo do comportamento de consumo sustentável. 
O estresse ambiental pode ser compreendido como um desequilíbrio nas condições ambientais de uma região ou ecossistema, gerando uma pressão ambiental, por exemplo, problemas com o $\mathrm{pH}$ da água, salinidade do solo, temperatura do ambiente ou poluição do ar (SUN; LIU; ZHAO, 2019).

Ainda segundo Sun, Liu e Zhao (2019, p. 489), “quanto maior o estresse ambiental, por exemplo, quanto mais grave a poluição que o consumidor percebe no ambiente, maior a pressão ambiental percebida, e os indivíduos mais dispostos a aumentar seu próprio consumo verde para melhorar o meio ambiente."

Sendo assim, consumidores sustentáveis que residam em cidades ou regiões com maiores níveis de desequilíbrios ou problemas ambientais são mais propensos a praticar tal comportamento pró-ambiental, tendo em vista contribuir com a recuperação do ambiente no qual estão inseridos, o que pode ser visto como uma oportunidade para que as empresas que focam no segmento de consumo sustentável deem especial atenção aos consumidores que residam em cidades e regiões com tais características (SUN; LIU; ZHAO, 2019).

\section{Taxa de Urbanização e Taxa de Penetração do Transporte Público}

Sun, Liu e Zhao (2019) validaram dois indicadores macrossociais como fatores determinantes e significativos do consumo sustentável, sendo estes a taxa de urbanização e a taxa de penetração do transporte público de uma localidade (país/Estado/cidade).

Quanto à taxa de urbanização, Sun, Liu e Zhao (2019, p. 490) afirmam que "tem um impacto significativo e positivo no consumo verde. Quanto maior a taxa de urbanização, mais consumidores urbanos existem. $\mathrm{O}$ ambiente urbano pode fornecer aos consumidores mais recursos verdes para promover o consumo verde."

Em relação à taxa de penetração do transporte público, Sun, Liu e Zhao (2019, p. 490) defendem que ela "tem um impacto significativo e positivo no consumo verde. 0 uso do transporte público está intimamente relacionado aos padrões de consumo das pessoas, e também é um tipo de consumo verde", e ainda que, em decorrência disso, "uma taxa crescente de penetração do transporte público pode promover a ocorrência do comportamento do consumidor verde."

Estas são mais algumas características das regiões mais propícias a possuir maior quantitativo de consumidores sustentáveis mais comprometidos com o comportamento de consumo sustentável, ou seja, além de um nível de stress ambiental alto (SUN; LIU; ZHAO, 2019), regiões mais propícias ao consumo sustentável também são aquelas que apresentam um maior nível de urbanização e de penetração do transporte público.

\section{Poluição Ambiental e Tendência Ambiental}

O modelo preditivo proposto e testado por Sun, Liu e Zhao (2019) ainda constatou influência significativa de dois outros fatores sob o comportamento de consumo sustentável: poluição ambiental e tendência ambiental. 
Em relação ao fator poluição ambiental, Sun, Liu e Zhao (2019, p. 490) explicam que "tem um efeito significativo no consumo verde. Com base na orientação da situação externa, quanto mais severos forem os níveis de poluição, maior a probabilidade de estimular as percepções dos consumidores sobre os riscos ambientais e consumo verde."

Quanto ao fator tendência ambiental, Sun, Liu e Zhao (2019, p. 490) pontuam que também é uma variável relevante para a explicação do consumo sustentável, pois as ações e hábitos dos consumidores são fortemente influenciados pelo ambiente que os circunda. Afirmam, ainda, que "se uma região como um todo tem uma forte tendência à proteção ambiental, isso influenciará os comportamentos ambientais individuais e promoverá o consumo verde."

\section{Nível de Religiosidade}

Os estudos de Mathras et al. (2016), Minton e Kahle (2016), Razzaq et al. (2018) e Minton et al. (2018), evidenciaram que o nível de religiosidade também é um fator determinante significativo do consumo sustentável.

De acordo com Minton et al. (2018, p. 660), "os resultados mostram que, em uma amostra dos Estados Unidos, país ocidental de maioria cristã, a religiosidade influencia positivamente o consumo sustentável." Os achados de Razzaq et al. (2018), com uma amostra do Paquistão, país oriental de maioria muçulmana, complementam os resultados de Minton et al. (2018), também identificando predição positiva e significativa do nível de religiosidade sob a propensão ao consumo sustentável. Ou seja, em âmbito global, quanto maior o nível de religiosidade maior a propensão ao consumo sustentável.

Os resultados e conclusões de Razzaq et al. (2018) complementam os achados da pesquisa de Minton et al. (2018) no sentido de que os próprios autores (Minton et al., 2018) assumem como limitação de sua pesquisa o fato de sua amostra ser composta apenas por cidadãos ocidentais e norte-americanos de maioria cristã, e sugerem, como complementação da pesquisa sobre a influência da religiosidade acerca do consumo sustentável, o desenvolvimento de pesquisa similar em países orientais com maioria muçulmana para verificar se o comportamento da relação entre os dois construtos se mantém ou se altera. Nesse sentido, os resultados do estudo empreendido por Razzaq et al. (2018) no oriente, com 295 respondentes, confirmam os achados da pesquisa de Minton et al. (2018) no ocidente, também constatando que, na atualidade, em um país de maioria muçulmana, a religiosidade é fator preditivo com significância estatística do consumo sustentável.

O fato de estudos realizados com amostras de consumidores ocidentais e orientais terem apontado no mesmo sentido e de que o nível de religiosidade influencia positivamente o comportamento de consumo sustentável, é especificamente importante para empresas que exploram o segmento de consumo sustentável e possuem operações globais, denotando que as empresas que focam em tal segmento, e atuam em qualquer parte do mundo, devem levar em consideração em suas estratégias de marketing o fato de que uma das características do seu público-alvo é que eles apresentam níveis mais altos de religiosidade do que a média. 


\section{Amor à Posse Material}

O amor pela posse material pode ser entendido como a natureza e o grau de apego emocional positivo do consumidor em relação a um objeto (DONG et al., 2018). De acordo com os resultados de seu estudo, o amor pela posse material é um dos fatores significativamente determinantes do consumo sustentável.

Os resultados da pesquisa de Dong et al. (2018) indicam que as necessidades de autonomia, afiliação e controle dos consumidores afetam significativamente o amor pela posse material, que, por sua vez, afeta o comportamento de consumo sustentável. Quanto maior o nível de amor pela posse material, maior a tendência ao consumo sustentável por parte dos consumidores.

\section{Influência da Família e dos Amigos}

A influência da família e dos amigos foi validada como um fator significativamente determinante do consumo sustentável por meio da operacionalização e teste do modelo preditivo proposto por Figueroa-García, García-Machado e Yábar (2018).

Segundo os resultados da pesquisa de Figueroa-García, García-Machado e Yábar (2018), quanto mais alguém recebe influência de amigos e familiares com alta predisposição ao consumo sustentável, mais predisposto ao consumo sustentável este indivíduo também estará.

Tal relação preditiva aponta para um desdobramento interessante. Se o contato com outros consumidores sustentáveis gera predisposição ao consumo sustentável, quanto mais consumidores adotarem práticas de consumo sustentável mais consumidores ainda tenderão a tornar-se também consumidores conscientes.

\section{Condições de Mercado}

Figueroa-García, García-Machado e Yábar (2018) também testaram em seu modelo preditivo a relação de causalidade entre o fator denominado condições de mercado e o consumo sustentável, tendo obtido evidência estatística significativa de que de fato o construto possui relação preditiva positiva em relação ao fenômeno dependente em estudo.

O fator denominado condições de mercado refere-se à percepção positiva de produtos sustentáveis pelos consumidores, ou seja, os resultados dos seus estudos revelaram que quanto mais positiva a percepção sobre os produtos sustentáveis por parte dos consumidores, mais estes estarão propensos a consumi-los, pagando, inclusive, preços mais altos para adquiri-los do que pagariam por produtos convencionais (FIGUEROA-GARCÍA; GARCÍA-MACHADO; YÁBAR, 2018).

\section{Acesso a Redes Sociais}

Apesar de os resultados obtidos por Sun, Liu e Zhao (2019), quanto a não significância estatística da taxa de penetração da internet sobre o consumo sustentável, serem alinhados aos de Wang e Hao (2018, p. 237), que identificaram em seu estudo que em 31 países a penetração da internet não influencia significativamente os comportamentos individuais de consumo sustentável, os resultados da pesquisa de Lien, Cao e Zhou (2017, p. 404) "sugerem que o comportamento de consumo sustentável é 
intrinsecamente influenciado pelo acesso dos indivíduos à tecnologia de rede social", validando estatisticamente o fator acesso às redes sociais como uma variável significativamente preditiva do consumo sustentável.

Tal influência advém da "possibilidade de compartilhar experiências ambientais com outros usuários e consultas sobre atividades ambientais favoráveis ao meio ambiente, como consumo de produtos verdes, reciclagem de resíduos e consumo de água e energia", o que, por sua vez, pode fazer "aumentar significativamente a conscientização ambiental e a escolha de produtos com credenciais ecológicas entre os outros usuários. Recomendação do usuário-usuário." (LIEN; CAO; ZHOU, 2017, p. 404).

Apesar da penetração da internet ter dado evidência de que não é um fator preditivo significativo do consumo sustentável, o acesso às redes sociais aparece como um preditor positivo deste tipo de comportamento.

A fim de avançar no sentido de atrair a atenção dos profissionais de mercado, mais especificamente da área do marketing, para os resultados desta pesquisa, para que tais resultados possam, de fato, gerar impacto no ambiente organizacional, no Quadro 2 é apresentada uma persona do consumidor sustentável, a partir dos diversos fatores determinantes do consumo sustentável identificados por meio da revisão de literatura empreendida neste estudo.

Vale salientar que a síntese apresentada foi construída tomando por base resultados de estudos realizados em diversas partes do mundo; portanto reflete um perfil global do consumidor sustentável, de forma que se sugere cautela e análise pormenorizada de regiões específicas do globo antes da sua utilização para embasamento das estratégias de marketing verde das corporações.

\section{Quadro 2 - Persona do consumidor sustentável a partir dos resultados da revisão de literatura}

Os consumidores sustentáveis são indivíduos que...

- Possuem alto nível de consciência ou preocupação ambiental;

- Estão atentos ao compromisso social das empresas, premiando aquelas que se mostram mais compromissadas;

- Possuem como traços marcantes da personalidade a extroversão e a agradabilidade;

- Têm maior grau de afeto ao ambiente;

- São mais susceptíveis ao sentimento de inveja;

- Apresentam maior preocupação com o bem-estar dos outros;

- Se preocupam com a efetividade do seu comportamento sustentável;

- São politicamente ativos;

- Possuem prioritariamente orientação política de esquerda;

- Planejam suas compras;

- São sensíveis à exposição a produtos verdes;

- Não só são conscientes, como praticam atitudes favoráveis ao meio ambiente;

- Residem em cidades ou regiões com maiores níveis de desequilíbrios ou problemas ambientais;

- Habitam em regiões com maior nível de urbanização e de penetração do transporte público;

- Vivem em localidades geralmente com altos índices de poluição ambiental;

- Moram em regiões ou territórios que promovem o consumo sustentável;

- São mais propensos à religiosidade;

- São apegados emocionalmente aos bens materiais;

- Recebem influência de amigos e familiares com alta predisposição ao consumo sustentável;

- Possuem percepção positiva sobre os produtos sustentáveis;

- Assíduos nas redes sociais.

Fonte: Elaboração própria, 2019. 


\section{CONSIDERAÇÕES FINAIS}

O notório crescimento do grupo de consumidores preocupado com a preservação do meio ambiente, a promoção do bem-estar da sociedade e o equilíbrio da economia (HAWN, CHATTERJI; MITCHELL, 2018), vêm chamando a atenção das empresas em âmbito mundial para a promoção de uma atuação mais sustentável (BULUT; ÇIMRIN; DOĞAN, 2017), a fim de conquistar o maior número de consumidores conscientes possível e, assim, se sobressair em meio a mercados cada vez mais competitivos (VERREYNNE et al., 2019).

Para que as empresas conquistem esses consumidores, entretanto, estas precisam ir além da identificação dos fatores determinantes do comportamento de consumo sustentável mais óbvios ou seminais, e buscar o contínuo reconhecimento de novos fatores potencialmente influenciadores de tal comportamento, para, assim, delinear meIhor as características do seu público-alvo, o que os motiva a realizar o ciclo do consumo sustentável e, dessa forma, possibilitar às empresas o desenvolvimento de uma melhor sintonia entre a sua imagem, produtos e serviços e as expectativas do cliente, e, consequentemente, maior eficiência nas vendas (STRAUGHAN; ROBERTS, 1999).

Nesse ínterim, dentre os 21 fatores identificados a consciência ambiental se sobressai, sendo apontada como a principal influenciadora do consumo sustentável entre os estudos analisados. Vários fatores, no entanto, menos intuitivos ou evidentes, também foram verificados, por exemplo: acesso às redes sociais; nível de religiosidade; taxa de penetração do transporte público; amor pela posse material; taxa de urbanização; emoções, entre outros, revelando, assim, um perfil bem mais detalhado dos consumidores sustentáveis, proporcionando, como argumenta Straughan e Roberts (1999), uma atualização importante dos fatores determinantes do consumo sustentável e das características desses consumidores para as empresas e órgãos públicos.

Por meio do potencial benéfico advindo do avanço teórico a ser promovido pelos resultados do presente estudo, esta pesquisa também traz importantes contribuições para o mercado e a sociedade.

Em relação à contribuição deste estudo para o mercado, Fraj e Martinez (2006) afirmam que "a segmentação do mercado ecológico, por meio de fatores influenciadores do consumo sustentável, é uma ação fundamental que permite às empresas o desenvolvimento de estratégias comerciais eficientes", e Minton et al. (2018, p. 661) complementam argumentando que "entender os valores de um consumidor [...] e sua influência no consumo de produtos e serviços relacionados à sustentabilidade pode ser fundamental para os planos de marketing de empresas e formuladores de políticas para promover práticas sustentáveis." (STRAUGHAN; ROBERTS, 1999, p. 575).

Quanto à contribuição da pesquisa para a sociedade e o meio ambiente, essa dá-se pelo fato de que, a partir dos resultados esperados, almeja-se proporcionar às empresas, aos órgãos públicos e às organizações do terceiro setor um melhor delineamento dos fatores motivadores do consumo sustentável, que, em posse de tais informações, poderão desenvolver estratégias com o intuito de serem mais assertivos no atendimento das demandas desse público, bem como fomentar o crescimento desse segmento, contribuindo, assim, com a solidificação do desenvolvimento sustentável. 
A principal limitação do presente estudo foi o fato de a revisão de literatura ter tomado por base apenas a Web of Science, que, embora seja uma das principais bases de dados e pesquisas científicas do mundo, abarcando grande parte do conteúdo científico relevante das mais diferentes áreas do conhecimento, possui suas limitações de abrangência.

Dessa forma, a primeira oportunidade de pesquisa futura, observada neste trabalho, é a realização de um esforço de revisão similar em outras bases de pesquisa relevantes para a área, como a Scopus, Scielo e Emerald, a fim de que outros trabalhos relevantes possam também ser considerados e, assim, possibilitar a construção de uma base teórica ainda mais sólida para a análise do comportamento de consumo sustentável, bem como uma persona ainda mais representativa desse público, aperfeiçoando, então, a formulação de estratégias de marketing ambiental.

Outra oportunidade de pesquisa apontada pela maioria dos pesquisadores lidos para a construção do framework apresentado neste relatório, é a proposição de modelos estatísticos com base em fatores explicativos emergentes, como os identificados neste trabalho e outros mais, capazes de explicar, de forma robusta, o novo perfil dos consumidores sustentáveis, haja vista não haverem modelos definitivos para tal intento, até mesmo pela característica mutante do comportamento dos consumidores, o que leva à necessidade de constante atualização dos modelos explicativos.

Segundo resultados da pesquisa de Semprebon et al. (2019), o campo carece principalmente de modelos estatísticos baseados em métodos que possam aferir relações diretas e indiretas de influência entre construtos diversos e o comportamento de consumo sustentável, bem como relações de mediação e moderação, como a Modelagem de Equações Estruturais - MEE.

\section{AGRADECIMENTOS}

O presente trabalho foi realizado com apoio da Coordenação de Aperfeiçoamento de Pessoal de Nível Superior - Brasil (Capes) - Código de Financiamento 001.

\section{REFERÊNCIAS}

ANTONETTI, P.; MAKLAN, S. An Extended Model of Moral Outrage at Corporate Social Irresponsibility. Journal of Business Ethics, v. 135, n. 3, p. 429-444, 2016. 10.1007/s10551-014-2487-y

BAMBERG, S.; MÖSER, G. Twenty years after Hines, Hungerford, and Tomera: a new meta-analysis of psycho-social determinants of pro-environmental behavior. Journal of Environmental Psychology, v. 27, n. 1, p. 14-25, 2007. DOI: 10.1016/j.jenvp.2006.12.002

BECK, C. G.; PEREIRA, R. C. F. Preocupação ambiental e consumo consciente: os meus, os seus e os nossos interesses. GeAS, v. 1, n. 2, p. 51-78, 2012. DOI: 10.5585/geas.v1i2.22

BEDANTE, G. N.; SLONGO, L. A. O comportamento de consumo sustentável e suas relações com a consciência ambiental e a intenção de compra de produtos ecologicamente embalados. In: ENCONTRO DE MARKETING DA ASSOCIAÇÃO NACIONAL DE PÓS-GRADUAÇÃO E PESQUISA EM ADMINISTRAÇÃO, 1., 2004, Porto Alegre. Anais [...]. Porto Alegre: Anpad, 2004.

BRENGMAN, M.; WILLEMS, K.; JOYE, Y. The Impact of In-Store Greenery on Customers. Psychology \& Marketing, v. 29, n. 11, p. 807-821, 2012. DOI: 10.1002/mar.20566

BRITO, L. M. P.; SILVA, N. E. A. S.; CASTRO, A. B. C.; NODARI, C. H.; SILVA, A. W. P. Knowledge management for the sustainable development of the semi-arid region in Northeastern Brazil. Ciência Rural, v. 49, n. 4, p. 2019. DOI: http://dx.doi.org/10.1590/0103-8478cr20180762 
BULUT, Z. A.; ÇIMRIN, F. K.; DOĞAN, O. Gender, generation and sustainable consumption: Exploring the behaviour of consumers from Izmir, Turkey. International Journal of Consumer Studies, v. 41, n. 6, p. 597604, 2017. DOI: 10.1111/ijcs.12371

CASTRO, A. B. C.; NODARI, C. H.; BRITO, L. M. P.; SILVA, A. W. P; SANTOS, H. C. C. Temáticas emergentes em gestão do conhecimento: um estudo bibliométrico. In: SEMINÁRIOS EM ADMINISTRAÇÃO, 21., 2018, São Paulo. Anais [...]. São Paulo: Semead.

CHAN, R. Y. K.; LAU, L. B. Y. Antecedents of green purchases: a survey in China. Journal of consumer marketing, v. 17, n. 4, p. 338-357, 2000. DOI: 10.1108/07363760010335358

CHEN, E. Y. I.; YEH, N.-C.; WANG, C. P. Conspicuous consumption: A preliminary report of scale development and validation. Advances in Consumer Research, v. 35, p. 686-687, 2008.

COELHO, A. L. A. L.; ARAÚJO, L. A. S.; SILVA, A. W. P. S.; SANTOS, H. C. C.; COELHO, C. Educação para sustentabilidade e gestão pública em uma escola estadual na cidade de João Pessoa-PB. Revista Interdisciplinar Científica Aplicada, v. 12, n. 4, p. 23-38, 2018a. Disponível em: https://rica.unibes.com.br/rica/issue/ view/68/showToc

COELHO, C.; MACEDO, J. D. F.; COELHO, A. L. A. L.; SILVA, A. W. P.; SANTOS, H. C. C. Desenvolvimento sustentável no acre: a execução orçamentária sob o enfoque da gestão ambiental. AOS, v. 7, n. 1, p. 93-116, 2018. DOI: http://dx.doi.org/10.17800/aos.v7i1.749

COELHO, A. L. A. L.; SANTOS, H. C. C.; SILVA, A. W. P.; COELHO, C.; OLIVEIRA, B. N. F. Sustainable watercolor: representative images of the conception of sustainability based on drawings by children and adolescents from João Pessoa. Revista de Administração da UFSM, v. 11, Ed. Especial Engema, p. 858-880, 2018. DOI: http://dx.doi.org/10.5902/1983465932612

COLARES, A. C. V.; MATTAR, P. Produtos verdes: análise das características potencialmente influenciadoras dos consumidores sustentáveis. Reunir, v. 6, n. 1, p. 37-55, 2016. DOI: 10.18696/reunir.v6i1.339

DE YOUNG, R. Expanding and evaluating motives for environmentally responsible behavior. Journal of Social Issues, v. 56, n. 3, p. 509-523, 2000. DOI: 10.1111/0022-4537.00181

DONG, X.; LI, H.; LIU, S.; CAI, C.; FAN, X. How does material possession love influence sustainable consumption behavior towards the durable products? Journal of Cleaner Production, v. 198, p. 389-400, 2018. DOI: 10.1016/j.jclepro.2018.07.054

ESPEJO, I. G.; VÁZQUEZ, A. N. The Emergence of the "Conscious Consumer": An Analysis of Political Participation through Purchasing Decisions. Rev. Esp. Investig. Sociol., n. 158, p. 59-78, 2017. DOI: 10.5477/ cis/reis.158.59

FIELDING, K. S.; MCDONALD, R.; LOUIS, W. R. Theory of planned behaviour, identity and intentions to engage in environmental activism. Journal of Environmental Psychology, v. 28, n. 4, p. 318-326, 2008. DOI: 10.1016/j.jenvp.2008.03.003

FIGUEROA-GARCÍA, E. C.; GARCÍA-MACHADO, J. J.; YÁBAR, D. Modeling the Social Factors That Determine Sustainable Consumption Behavior in the Community of Madrid. Sustainability, v. 10, n. 8, p. 2811-2827, 2018. DOI: $10.3390 /$ su10082811

FRAJ, E.; MARTINEZ, E. Influence of personality on ecological consumer behaviour. Journal of Consumer Behaviour, v. 5, n. 3, p. 167-181, 2006. DOI: 10.1002/cb.169

FRAJ, E.; MARTINEZ, E. Ecological consumer behavior: an empirical analysis. International Journal of Consumer Studies, v. 31, n. 1, p. 26-33, 2007. DOI: 10.1111/j.1470-6431.2006.00565.x

GARCÍA, M. S. A.; DURANA, C. A. G.; ABANDO, J. C.; MOLINA, A. V. El Consumidor Ecológico: un modelo de comportamiento a partir de la recopilación y análisis de la evidencia empírica. Capital Humano, v. 13, n. 67, p. 41-54, 2003.

GONÇALVES-DIAS, S. L. F.; TEODÓSIO, A. S. S.; CARVALHO, S.; SILVA, H. M. R. Consciência ambiental: um estudo exploratório sobre suas implicações para o estudo de Administração. RAE-Eletrônica, v. 8, n. 1, 2009. DOI: 10.1590/S1676-56482009000100004

HAWN, O.; CHATTERJI, A. K.; MITCHELL, W. Do investors actually value sustainability? New evidence from investor reactions to the Dow Jones Sustainability Index (DJSI), Strat. Manag. J., v. 39, n. 4, p. 949-976, 2018. DOI: $10.1002 / \mathrm{smj} .2752$

IPCC. INTERGOVERNMENTAL PANEL ON CLIMATE CHANGE. Climate Change 2014: Synthesis Report. Contribution of Working Groups I, II and III to the Fifth Assessment Report of the Intergovernmental Panel on Climate Change, 2014. Disponível em: https://www.ipcc.ch/pdf/assessment-report/ar5/syr/SYR_AR5_FINAL_full_wcover.pdf. Acesso em: 2 mar. 2019.

JOSHI, Y.; RAHMAN, Z. Investigating the determinants of consumers' sustainable purchase behaviour. Sustainable Production and Consumption, v. 10, p. 110-120, 2017. DOI: 10.1016/j.spc.2017.02.002 
KHAN, M. I.; KHAN, S.; HALEEM, A.; JAVAID, M. Prioritising Barriers towards Adoption of Sustainable Consumption and Production Practices using TOPSIS. IOP Conf. Ser.: Mater. Sci. Eng., v. 404, 2018. DOI: 10.1088/1757-899X/404/1/012011

KARMARKAR, U. R.; BOLLINGER, B. BYOB: how bringing your own shopping bags leads to treating yourself and the environment. Journal of Marketing, v. 79, n. 4, p. 1-15, 2015. DOI: 10.1509/jm.13.0228

LAGES, N. S.; VARGAS NETO, A. Mensurando a consciência ecológica do consumidor: um estudo realizado na cidade de Porto Alegre. In: ENCONTRO NACIONAL DA ANPAD, 26., 2002, Salvador. Anais [...]. Salvador: Anpad, 2002.

LATIF, S. A.; OMAR, M. S.; BIDIN, Y. H.; AWANG, Z. Role of environmental knowledge in creating pro environmental residents. Procedia-Social and Behavioral Sciences, v. 105, p. 866-874, 2013. DOI: 10.1016/j. sbspro.2013.11.088

LIEN, C.-H.; CAO, Y.; ZHOU, X. Service quality, satisfaction, stickiness, and usage intentions: An exploratory evaluation in the context of WeChat services. Computers in Human Behavior, v. 68, p. 403-410, 2017. DOI: 10.1016/j.chb.2016.11.061

MALONEY, M. P.; WARD, M. P.; BRAUCHT, G. N. A revised scale for the measurement of ecological attitudes and knowledge. American Psychologist, v. 30, n. 7, p. 787-790, 1975. DOI: 10.1037/h0084394

MARQUINA, P.; MORALES, C. E. The influence of CSR on purchasing behaviour in Peru and Spain. International Marketing Review, v. 29, n. 3, p. 299-312, 2012. DOI: 10.1108/02651331211229778

MATHRAS, D.; COHEN, A. B.; MANDEL, N.; MICK, D. G. The effects of religion on consumer behavior: A conceptual framework and research agenda. Journal of Consumer Psychology, v. 26, n. 2, p. 298-311, 2016. DOI: 10.1016/j.jcps.2015.08.001

MAZAR, N.; ZHONG, C. B. Do green products make us better people? Psychological Science, v. 21, n. 4, p. 494-498, 2010. DOI: 10.1177/0956797610363538

MINTON, E. A.; KAHLE, L. R. Religion and consumer behaviour. In: JANSSON-BOYD, C. V.; ZAWISZA, M. J. (ed.). International handbook of consumer psychology. New York-EUA: Routledge, p. 292-311, 2016.

MINTON, E. A.; XIE, H. J.; GUREL-ATAY, E.; KAHLE, L. R. Greening up because of god: The relations among religion, sustainable consumption and subjective well-being. International Journal of Consumer Studies, v. 42, n. 6, p. 655-663, 2018. DOI: 10.1111/ijcs.12449

MOURA, L. K. B.; MESQUITA, R. F.; MOBIN, M.; MATOS, F. T. C.; MONTE, T. L.; LAGO, E. C.; FALCÃO, C. A. M.; FERRAZ, M. A. A. L.; SANTOS, T. C.; SOUSA, L. R. M. Uses of 16 Bibliometric Techniques in Public Health Research. Iranian Journal of Public Health, v. 46, n. 10, p. 1.435-1.436, 2017.

PAUL, J.; MODI, A.; PATEL, J. Predicting green product consumption using theory of planned behavior and reasoned action. Journal of Retailing and Consumer Services, v. 29, p. 123-134, 2016. DOI: 10.1016/j.jretconser.2015.11.006

RAZZAQ, Z.; RAZZAQ, A.; YOUSAF, S.; HONG, Z. The Impact of Utilitarian and Hedonistic Shopping Values on Sustainable Fashion Consumption: The Moderating Role of Religiosity. Global Business Review, v. 19, n. 5, p. 1.224-1.239, 2018. DOI: 10.1177/0972150918777947

RIBEIRO, J. A.; VEIGA, R. T. Proposição de uma escala de consumo sustentável. R. Adm., v. 46, n. 1, p. 4560, 2011. DOI: 10.5700/rausp0997

ROBERTS, J. A. Green consumers in the 1990s: profile and implications for advertising. Journal of Business Research, v. 36, n. 3, p. 217-231, 1996. DOI: 10.1016/0148-2963(95)00150-6

SEMPREBON, E.; MANTOVANI, D.; DEMCZUK, R.; MAIOR, C. S.; VILASANTI, V.; MANTOVANI, D.; DEMCZUK, R. Green consumption: a network analysis in marketing. Marketing Intelligence \& Planning, v. 37, n. 1, p. 18-32, 2019. DOI: 10.1108/MIP-12-2017-0352

SILVA, A. W. P.; COELhO, A. L. A. L., SANTOS, H. C. C.; VEIGA NETO, A. R.; CASTRO, A. B. C.; El-AOUAR, W. A. Education principles and practises turned to sustainability in primary school. Environ Dev Sustain, 2019. DOI: 10.1007 / s10668-019-00505-2

SILVA, A. W. P.; OlIVEIRA, B. N. F.; SIQUEIRA, E. S.; COELHO, A. L. A. L.; SANTOS, H. C. C. Análise das políticas de sustentabilidade e responsabilidade social como diferencial competitivo para as empresas. $O$ Eco da Graduação, v. 3, n. 1, p. 91-106, 2018. Disponível em: http://ecodagraduacao.com.br/index.php/ ecodagraduacao/article/view/44

SILVA, B. G.; SANTOS, H. C. C.; SILVA, A. W. P.; COELHO, A. L. A. L.; OlIVEIRA, B. N. F.; PELLIZZONI, L. N.; MOURA NETO, J. S.; SABINO, F. H. P. S. Análise do discurso de sustentabilidade da Empresa Samarco a partir de materiais visuais. Facear, v. 1, n. 7, 2018. Disponível em: http://revista.facear.edu.br/artigo/\$/ analise-do-discurso-de-sustentabilidade-da-empresa-samarco-a-partir-de-materiais-visuais

SILVA, A. W. P.; SIQUEIRA, E. S. A internalização dos princípios da norma ISO 26000: o caso da Petrobras. Holos, v. 1, ano 31, p. 227-243, 2015. DOI: https://doi.org/10.15628/holos.2015.2198 
SILVA, V. H. M.; SILVEIRA-MARTINS, E.; OTTO, I. M. Mensuração da consciência ambiental dos consumidores: proposta e validação de escala. Revista de Administração da UFSM, v. 10, p. 63-78, 2017. DOI: 10.5902/1983465925201

SOMERVILLE, M.; WILLIAMS, C. Sustainability education in early childhood: An updated review of research in the field. Contemporary Issues in Early Childhood, v. 16, n. 2, p. 102-117, 2015. DOI: 10.1177/1463949115585658

STONE, G.; BARNES, J. H.; MONTGOMERY, C. ECOSCALE: The scale for the measurement of environmentally responsible consumers. Psychology \& Marketing, v. 12, n. 7, p. 595-612, 1995. DOI: 10.1002/ mar.4220120704

STRAUGHAN, R. D.; ROBERTS, J. A. Environmental segmentation alternatives: a look at green consumer behavior in the new millennium. Journal of Consumer Marketing, v. 16, n. 6, p. 558-575, 1999. DOI: $10.1108 / 07363769910297506$

SUN, Y. H.; LIU, N. N.; ZHAO, M. Z. Factors and mechanisms affecting green consumption in China: A multilevel analysis. Journal of Cleaner Production, v. 209, p. 481-493, 2019. DOI: 10.1016/j.jclepro.2018.10.241 TARKIAINEN, A.; SUNDQVIST, S. Subjective norms, attitude and intentions of Finnish consumers in buying organic food. Brit Food J., v. 107, n. 11, p. 808-822, 2005. DOI: 10.1108/00070700510629760

ÜLKÜ, M. A.; HSUAN, J. Towards sustainable consumption and production: Competitive pricing of modular products for green consumers. Journal of Cleaner Production, v. 142, p. 4230-4242, 2017. DOI: 10.1016/j.jclepro.2016.11.050

VERREYNNE, M. L.; WILLIAMS, A. M.; RITCHIE, B. W.; GRONUM, S.; BETTS, K. S. Innovation diversity and uncertainty in small and medium sized tourism firms. Tourism Management, v. 72, p. 257-269, 2019. DOI: 10.1016/j.tourman.2018.11.019

WANG, Y.; HAO, F. Does Internet penetration encourage sustainable consumption? A cross-national analysis. Sustainable Production and Consumption, v. 16, p. 237-248, 2018. DOI: 10.1016/j.spc.2018.08.011 WANG, C.; GHADIMI, P.; LIM, M. K.; TSENG, M. L. A literature review of sustainable consumption and production: A comparative analysis in developed and developing economies. Journal of Cleaner Production, v. 206, p. 741-754, 2019. DOI: 10.1016/j.jclepro.2018.09.172

ZAREIE, B.; NAVIMIPOUR, N. J. The impact of electronic environmental knowledge on the environmental behaviors of people. Computers in Human Behavior, v. 59, p. 1-8, 2016. DOI: 10.1016/j.chb.2016.01.025 